\title{
Statuts des langues et éducation de base aux
} Comores

Statuses of languages and basic education in the Comoros

Situación de las lenguas y educación básica en las Comores

\section{Aurélie Chauvet}

\section{(2) OpenEdition}

Journals

Édition électronique

URL : https://journals.openedition.org/ries/4499

DOI : 10.4000/ries.4499

ISSN : 2261-4265

Éditeur

France Education international

Édition imprimée

Date de publication : 1 décembre 2015

Pagination : 77-84

ISSN : 1254-4590

Référence électronique

Aurélie Chauvet, "Statuts des langues et éducation de base aux Comores », Revue internationale d'éducation de Sèvres [En ligne], 70 | décembre 2015, mis en ligne le 01 décembre 2017, consulté le 01 juillet 2021. URL : http://journals.openedition.org/ries/4499; DOI : https://doi.org/10.4000/ries.4499

(c) Tous droits réservés 


\section{Statuts des langues et éducation de base aux Comores}

\section{Aurélie Chauvet \\ Enseignante et doctorante}

La place attribuée à chaque langue dans les structures pédagogiques a un rôle indéniable. En donnant à chacune d'elles la place appropriée, il est possible de contribuer à une amélioration des apprentissages des élèves. Nous analyserons ici comment les politiques linguistiques éducatives, aux Comores, pourraient être mises au service de la réussite scolaire.

Les Comores ont trois langues officielles : le français - le pays a été colonie française jusqu'à son indépendance en 1975 -, l'arabe - le pays est de confession musulmane - depuis 1985 et le shikomori depuis 1992. Un décret donnant lieu à la standardisation de cette dernière est paru en 2009. La langue comorienne avait déjà connu diverses propositions de standardisation de l'écriture : en caractères arabes (Kamar-Eddine) et en caractères latins dans les années 1977-1978, sous le régime d'Ali Soilihi, qui lança une campagne d'alphabétisation en comorien.

Ces trois langues sont utilisées dans des sphères coïncidant en partie seulement : le français est la langue de l'écrit, celle de l'administration, celle de la très grande majorité des ouvrages scolaires à partir du primaire. L'arabe est la langue religieuse, la langue d'enseignement des écoles coraniques et donc la première langue dans laquelle sont alphabétisés les enfants comoriens, ces derniers intégrant ces écoles lorsqu'ils sont âgés de 3 à 5 ans, avant l'entrée à l'école primaire. Le shikomori, enfin et surtout, est la langue première des Comoriens, celle qui apparaît dans toutes les sphères sociales mais dont la reconnaissance tardive en tant que langue officielle à côté du français et de l'arabe ne favorise pas encore sa mise en valeur ni l'acquisition d'un véritable prestige aux yeux des Comoriens eux-mêmes. En effet, les Comoriens continuent à établir, au quotidien, un rapport hiérarchique entre le français et le shikomori. Celui-ci, pourtant bien vivant, reste la langue familière, familiale et tend à peu sortir de ces sphères d'utilisation. De plus, l'existence de nombreuses alternances codiques avec le français témoigne de la représentation modeste que se font les Comoriens de leur propre langue. Elle n'est utile « que » pour communiquer au quotidien. Ce rapport diglossique que le shikomori entretient avec le français est d'autant plus visible dans une perspective scolaire : s'il n'est pas perçu de manière évidente comme langue enseignante et enseignée par les Comoriens, il est surtout pour l'instant peu présent concrètement dans les écoles, où les outils pédagogiques pour l'enseignement du et en comorien sont en cours de création. Ainsi, deux aspects amoindrissent la place de la langue 
comorienne dans le système éducatif : ses représentations sociales et sa faible visibilité écrite par le biais de supports didactiques.

L'existence de supports didactiques attrayants et motivants pourrait avoir un impact positif sur le premier aspect relevé. Or le contexte économique rend difficile le financement de tels outils pédagogiques, tant par les familles que par l'État : le pays occupait en effet la $169^{\mathrm{e}}$ place sur 186 avec un Indicateur de développement humain (IDH) de 0,429 en 2013. De plus, entre 1980 et 2010, la population des trois îles de l'Union des Comores a plus que doublé, passant de 329000 habitants à 691000 habitants, ce qui ajoute à la complexité de la gestion des structures éducatives.

Le taux brut de scolarisation est très faible en préscolaire $(5,1 \%$ en 2009-2010), au-delà de $100 \%$ pour le primaire (chiffre cependant probablement surestimé, et prenant en compte les redoublements, plutôt fréquents dans le système éducatif); ce taux descend à $52 \%$ au premier cycle du secondaire.

Cependant, ce taux de scolarisation ne signifie pas qu'un grand nombre d'enfants finissent le cycle. Il est avant tout révélateur d'une capacité d'accueil. En effet, le taux d'achèvement du cycle se situe à un niveau nettement inférieur à l'accès. Le taux de rétention est de $75 \%$, ce qui signifie qu'un quart des élèves entrant en CP1 ne termineront pas leur cycle jusqu'au CM2, mais reste cependant au-dessus de la moyenne des pays à faible revenu de l'Afrique subsaharienne. Enfin, 63,2 \% d'une classe d'âge termine le cycle primaire : la différence avec le taux global de $75 \%$ de rétention s'explique par davantage d'abandons en fin de cycle, les deux dernières années : les enfants sont en effet déjà âgés, souvent en raison de redoublements fréquents, dus à des situations d'échec scolaire.

Nous soulignerons ici l'importance du cycle primaire, d'autant plus crucial que seuls trois quarts des $62,3 \%$ d'élèves cités précédemment terminant le cycle primaire commencent ensuite le cycle secondaire. Or l'abandon en fin de cycle, d'après le Rapport d'État du système éducatif comorien, n'est apparemment pas dû à des différenciations sociales mais plutôt aux dispositifs pédagogiques inadaptés.

Notre contribution tâchera de déterminer en quoi le statut des langues et la valorisation du rôle de chacune peuvent avoir un impact positif sur l'amélioration du système éducatif aux Comores et pourraient contribuer à enrayer l'abandon de la scolarité et l'échec scolaire. Nous tenterons de cerner les faiblesses actuelles tout en proposant des pistes de réflexion pour rendre les politiques linguistiques en lien avec l'éducation de base plus performantes aux Comores.

\section{L'ENSEIGNEMENT PRÉÉLÉMENTAIRE : UNE TENTATIVE DE MISE EN VALEUR DE LA LANGUE PREMIÈre}

Aujourd'hui, l'éducation de base au niveau préélémentaire aux Comores se réalise à travers les écoles coraniques : de 3 à 5 ans, les élèves suivent des 
enseignements dont l'objectif principal est la connaissance et la lecture du Coran ${ }^{1}$. La première alphabétisation des enfants se fait donc en langue arabe, l'une des trois langues officielles; cependant cette alphabétisation n'a à ce jour pas d'autre vocation que celle de lire les textes sacrés. Les enseignements concernant la religion sont diffusés en comorien. Ainsi, la place de la langue arabe est très particulière en préélémentaire : elle est avant tout la langue associée aux textes sacrés et par là même, la langue sacrée, ce qui lui confère un prestige certain. Elle n'est cependant pas, à l'heure actuelle, un outil de diffusion des savoirs, n'étant ni langue administrative ni langue d'enseignement aux niveaux élémentaire et préélémentaire. En revanche, d'un point de vue symbolique, elle est la langue qui permet aux Comores de renforcer leur appartenance au monde arabo-musulman ${ }^{2}$.

Chamanga et Gueunier (1977) soulignent cependant qu'il est dommage de ne pas s'appuyer sur cette connaissance de la structure de la langue arabe qu'ont la plupart des enfants comoriens : en effet, ils expliquent que la formation des mots en arabe a certaines similitudes avec la formation des mots en comorien, ce qui permettrait de faire des liens d'une langue à l'autre. Ils évoquent aussi l'importance des apports lexicaux arabes en comorien, ainsi que l'introduction constante, en comorien, de lexèmes arabes sous la forme de néologismes. Ainsi, cette première connaissance de l'arabe pourrait jouer un rôle dans la construction de la grammaire de l'enfant, mais l'utilisation de la langue arabe est pour l'instante circonscrite au domaine religieux.

En 2007, un projet de réforme de ces écoles coraniques a vu le jour. Actuellement, plusieurs écoles pilotes mettent en place ce projet de réforme, rendu officiel par la loi d'orientation de 2014 sur l'éducation Cependant, dans les faits, un très grand nombre d'élèves poursuivent leur éducation préélémentaire dans les écoles coraniques traditionnelles.

Les écoles coraniques rénovées, à l'initiative du ministère et financées par l'Unicef et des ONG, ont renforcé et amélioré les contenus éducatifs de cet enseignement préscolaire, jouissant jusqu'à présent d'une certaine autonomie en matière de contenus d'enseignement. L'une des composantes du programme est l'introduction de la langue comorienne.

La langue d'enseignement de ces trois années d'éducation est la langue nationale, le shikomori. L'enfant doit savoir parler sa langue, celle de la langue de tous les jours, de la famille, avant de s'exprimer dans d'autres langues.

On note ici une réelle prise de conscience par les décideurs de l'importance de la maîtrise de la langue maternelle pour que l'apprentissage des autres langues soit efficace. Cela rejoint les recommandations de l'Unesco, émises dès 1953 :

II est évident que le meilleur support pour enseigner à un enfant est sa langue maternelle. Psychologiquement, c'est le système des signes compris qui marche automatiquement dans son esprit pour s'exprimer et comprendre.

1. Les objectifs évoluent à partir de 2007 à travers les écoles coraniques rénovées.

2. Les Comores sont membres de la Ligue arabe. 
Sociologiquement, c'est un moyen de s'identifier aux membres de la communauté à laquelle il appartient. Sur le plan pédagogique, il apprend plus rapidement par son biais que par celui d'un support linguistique qui ne lui est pas familier.

Il est précisé dans ces mêmes programmes que la langue comorienne doit être la référence, et que les autres langues, le français et l'arabe, introduites également dès la première année, doivent être des langues secondes pour l'apprenant. Le programme insiste sur le fait que l'élève doit surtout être familiarisé avec les sons de ces langues, sans que cela interfère avec les activités proposées dans sa langue maternelle. Il est ainsi précisé que la connaissance du shikomori est une priorité :

[...] l'élève doit savoir parler sa langue, celle de la vie de tous les jours, de la famille, avant de s'exprimer dans les autres langues.

Dans le Paquet minimum pour l'enseignement préélementaire coranique, les limites de l'utilisation du shikomori comme langue d'enseignement étaient cependant soulignées ; la langue n'était en effet pas encore standardisée en 2007, ce qui rendait compliquée son utilisation effective. Sa standardisation, première étape nécessaire à l'utilisation du comorien comme outil scolaire a eu lieu en 2009. La réforme des écoles coraniques a sans doute permis que cette première étape se produise assez rapidement après l'élaboration du Paquet minimum. Cependant le décret établissant cette standardisation fait état d'une langue comorienne unique, avec des variantes; ce décret propose une standardisation, mais ne choisit aucune des variantes, car cela, bien entendu, provoquerait des débats politiques autour de la création d'une langue comorienne unique, ce qui n'est pas à l'ordre du jour. Ainsi, la coexistence actuelle des trois variantes - le shiNgazidja (parlé à Grande Comore), le shiNwali (parlé à Mohéli), le shiNdzuani (parlé à Anjouan) - dans le système éducatif rend plus complexe la création d'outils pédagogiques, puisqu'ils ne peuvent être communs aux trois îles.

L'étape suivante concernait la mise en place d'une formation des formateurs et la création d'outils pédagogiques. Un plan de formation quadriennal, dont l'année zéro était programmée en 2009 a été établi en ce sens ${ }^{3}$. En 2009 ont effectivement eu lieu des ateliers de sensibilisation à destination des inspecteurs, des conseillers pédagogiques et des journalistes. L'objectif premier était d'enclencher une prise de conscience des structures linguistiques de la langue comorienne : connaître le mode de fonctionnement de sa langue pour mieux enseigner la langue et dans la langue.

Les étapes suivantes prévues (an II et an III) pour les années suivantes n'ont pas encore été mises en place. Elles consistaient essentiellement en l'élaboration de matériel pédagogique et en l'utilisation de manuels pilotes

3. M.A. Chamanga, Le comorien dans le système éducatif, rapport de mission (23 juin-31 juillet 2008) : Annexe III : Le programme des formations et d'élaboration de manuels didactiques? 
en cours préparatoire, puis en une phase d'amélioration desdits manuels. L'introduction du comorien se trouve pour l'instant freinée par ce manque de production d'outils pédagogiques, production rendue compliquée, d'une part, comme nous l'avons dit, par l'absence d'une véritable langue unique et d'autre part, par le financement de cette production. La création de supports communs avec des déclinaisons spécifiques pourrait cependant s'envisager, grâce à une étroite collaboration entre enseignants des trois îles.

Selon l'inspection du ministère de l'éducation nationale ${ }^{4}$, les écoles coraniques rénovées ont été intégrées dans le préscolaire du système éducatif, sous l'appellation « enseignement coranique rénové. De plus, cet enseignement préscolaire sera réduit à deux ans, tout en intégrant en amont une éducation parentale, en comorien, axée sur le développement et la santé de l'enfant.

\section{LE PROGRAMME D'APPUI AU FRANÇAIS : RENFORCEMENT DES COMPÉTENCES DANS LA LANGUE D'ENSEIGNEMENT}

Les Comores, en tant qu'ancienne colonie française indépendante depuis 1975, ont le français en héritage. C'est donc un legs à métamorphoser, à considérer non plus comme une langue de domination mais au contraire comme une langue de la modernité, ouvrant une porte sur le monde francophone - 220 millions de locuteurs et 77 États -, une langue technique aussi, donnant accès aux études supérieures au sein de toute la francophonie.

Cependant, la position du français aux Comores est actuellement fragilisée : du point de vue des représentations, la relation au français et à ce qui est français reste ambiguë : Mayotte, la quatrième île de l'archipel, département français depuis 2011, reste revendiquée. Les Comoriens demeurent ainsi partagés entre une nostalgie - dévalorisant la culture comorienne très souvent et l'aptitude du pays à se prendre en main - de la colonisation française, et l'idée d'un pays " incomplet », à qui il manquerait Mayotte. La culture comorienne est donc imprégnée d'une francophonie réelle mais complexe : celle d'un héritage colonial confronté à une situation politique actuelle ambiguë, dont Mayotte est le symbole récurrent. La représentation linguistique du français ne peut que souffrir de cette présence vive du passé. Et en effet, entre rêve et réalité, les rapports France/Comores, régis par cette monnaie d'échange politique permanente qu'est Mayotte, ne contribuent sans doute pas à rendre serein le rapport à la langue française, pourtant le meilleur instrument linguistique actuel susceptible de permettre aux Comores d'accéder à la modernité économique.

4. Entretien de l'auteur avec Amroine Darkaoui, inspecteur, le 18 juin 2014, Moroni, ministère de l'éducation nationale. 
Ainsi, le programme «Appui au français aux Comores» (AFC) a été lancé par la coopération française et mis en œuvre par le Centre international d'études pédagogiques $(\mathrm{CIEP})^{5}$ suite à un constat alarmant : la dégradation de la maîtrise du français, langue d'enseignement, dans les écoles comoriennes, alors que l'enseignement s'est toujours déroulé dans cette langue, du primaire au supérieur. L'objectif principal du projet est de contribuer à l'amélioration durable de la maîtrise du français par le corps enseignant. Le programme propose pour cela deux composantes :

- le renforcement de la formation initiale et continue des enseignants à tout niveau du système éducatif ;

- la création d'un environnement francophone autour du livre et du multimédia.

On s'aperçoit, à la lecture du compte-rendu de la réunion du Comité de pilotage du projet du 2 juin $2014^{6}$, que ce programme d'appui au français aux Comores est étroitement lié à un appui au renforcement de l'éducation de base dans le pays.

Ainsi, les premiers résultats évoqués concernent les actions de formation réalisées auprès des enseignants (800 enseignants formés depuis avril 2003 dans les alliances françaises, à raison de 100 heures par enseignant de mise à niveau dans la langue) mais aussi des actions visant à stabiliser et à structurer cette formation, avec l'élaboration, entre autres, d'une maquette des formations aux métiers de l'éducation. Parallèlement, de nombreuses formations de cadres de l'audiovisuel ont été organisées. Ces différentes actions de formation en français visent à donner davantage de poids et d'organisation aux enseignements en français et à la langue française dans les médias. Cependant, dans le même temps, le bagage technique que ces actions diffusent doit aussi contribuer à l'amélioration de l'éducation de base, en offrant aux formateurs comme aux cadres des médias des outils de formation pour l'éducation des élèves et de la population dans son ensemble.

\section{L'ÉDUCATION DE BASE POUR ADULTES ET JEUNES DÉSCOLARISÉS}

\section{Maeecha : l'alphabétisation des adultes en comorien}

Maeecha, mouvement associatif pour l'éducation et l'égalité des chances, travaille avec l'ensemble des acteurs de l'éducation aux Comores. Cette organisation non gouvernementale dynamique financée par l'Unicef, et créée en 2005, est l'initiatrice d'actions au sein des écoles comoriennes, pendant les vacances 
scolaires ou en cours du soir ; cette initiative a conduit à l'apparition d'une demande également d'alphabétisation en français. Ici surgit une problématique intéressante : cette demande formulée par les apprenants eux-mêmes pousse les responsables de l'association à s'interroger sur l'articulation entre l'apprentissage $\mathrm{du}$ shikomori et l'apprentissage du français.

L'association intervient également dans les villages, à destination d'adultes : ce programme, dans le cadre de l'orientation et des objectifs du Plan directeur et de l'Éducation pour tous (EPT) de grande envergure, mené par le centre d'alphabétisation et d'enseignement à distance Alfad, touche les trois îles. Il part, en particulier, du constat d'échec de l'alphabétisation à l'école. On estime en effet que 49,7\% de la population adulte de 15 à 45 ans ne saurait pas lire, ce qui représente environ 133000 individus au plan national. Cependant, le programme insiste sur la nécessaire amélioration de l'éducation de base, car un enfant convenablement alphabétisé sera un futur parent qui valorisera l'école et l'apprentissage des bases auprès de ses enfants.

\section{Programme d'alphabétisation en français d'adultes à l'Alliance française}

Un professeur exerçant à l'Alliance française depuis 1999 à raison de dix heures par semaine, enseigne à des adultes non scolarisés ou déscolarisés au niveau primaire. L'enseignant évoque comme raison première d'inscription à ce cours un manque d'intégration. On peut avancer l'hypothèse que ce manque d'intégration vient plutôt d'un sentiment d'exclusion vis-à-vis des supports écrits, en français exclusivement, que d'un manque d'intégration linguistique dans un contexte local où la langue utilisée est la langue première du public. De même, la langue française a été, dans le cadre de certains de ces ateliers d'alphabétisation, une passerelle pour l'acquisition d'autres savoirs de base : le professeur en est venu à proposer du calcul, de la menuiserie... On voit bien que la langue est un support essentiel, dans ces cas concrets, pour accéder à l'ensemble des savoirs.

\section{RÉUSSITES ET LIMITES DES POLITIQUES LINGUISTIQUES ACTUELLES DANS L'ÉDUCATION DE BASE}

Les politiques linguistiques éducatives actuelles tiennent pleinement compte de la place que les langues doivent occuper dans le système éducatif. Il est important de souligner que chaque langue officielle doit être maintenue et valorisée dans sa fonction, car chacune constitue un pilier essentiel de la construction identitaire de l'élève. Le shikomori d'abord, car c'est sa langue première : sa valorisation doit être donc essentielle au préscolaire, mais il doit être maintenu tout le long de la scolarité primaire, sur un pied d'égalité avec le 
français, introduit d'abord à hauteur de $10 \%$ au début du cycle, puis à $50 \%$ en fin de cycle. La langue française doit occuper sa juste place : celle de langue de la modernisation et de l'accès aux savoirs, en particulier écrits, et non plus en tant que langue dominante. L'arabe enfin, langue structurante car langue religieuse, devra aussi conserver sa place au préscolaire, faisant partie de l'identité sociale et communautaire de l'enfant. La connaissance de l'alphabet arabe par les élèves est également un élément dont il faut avoir conscience : c'est une richesse à utiliser et à prendre en compte dans la construction des apprentissages de base.

Nous noterons aussi qu'un ensemble d'actions et d'outils en matière d'alphabétisation pour adultes en shikomori a été mené à bien : le travail effectué dans ce domaine est pour l'instant plus novateur et plus dynamique que ce qui est effectué dans les écoles. L'articulation entre le comorien et le français est d'ailleurs déjà envisagée par l'association Maeecha, puisque cela répondrait à une demande des élèves alphabétisés en comorien. Les outils créés pourraient inspirer l'éducation de base, en manque de matériaux pédagogiques articulant les deux langues, voire être utilisés dans certains cas. Un travail collaboratif entre enseignants dans l'éducation de base et enseignants d'adultes alphabétisés, concernant ces outils bilingues, pourrait ainsi être une piste de travail, parmi d'autres, pour construire ensemble un nouveau modèle éducatif linguistique, garant d'une meilleure réussite scolaire.

\section{BiBLIOGRAPHIE}

CHAMANGA M.A., GUEUNIER N.J. (1977) : «Recherches sur l'instrumentalisation du comorien : problèmes d'adaptation lexicale d'après la version comorienne de la loi du 23 novembre 1974) ", Cahiers d'études africaines, nº 66, vol. 17, p. 213-239.

CHAMANGA M.A. (2008) : Le comorien dans le système éducatif, rapport de mission : Annexe III : Le programme des formations et d'élaboration de manuels didactiques, 23 juin-31 juillet, Ministère de l'éducation (non publié).

COMORES (2012): Diagnostic du système éducatif comorien pour une politique nouvelle dans le cadre de l'EPT, Rapport d'État du système éducatif comorien, février, 172 p. [http://goo.gl/QKRYNJ]

LAFON P. (2007) : «Le système Kamar-Eddine : une tentative originale d'écriture du comorien en graphie arabe ", Ya Mkobe, n 14-15, janvier, p. 29-48 [http://llacan.vjf. cnrs.fr/publications/Kamar-Eddine.pdf]

UNESCO (1953): The use of vernacular languages in education, Unesco (Monographs on education). [http://goo.gl/624LnQ] 